\title{
Analysis of somitogenesis using multiphoton laser scanning microscopy (MPLSM)
}

Mary E. Dickinson, Kenneth J. Longmuir, Scott E. Fraser

Mary E. Dickinson, Kenneth J. Longmuir, Scott E. Fraser, "Analysis of somitogenesis using multiphoton laser scanning microscopy (MPLSM)," Proc. SPIE 4262, Multiphoton Microscopy in the Biomedical Sciences, (24 April 2001); doi: $10.1117 / 12.424570$

EPIE Event: BiOS 2001 The International Symposium on Biomedical Optics, 2001, San Jose, CA, United States 
Invited Paper

\title{
Analysis of Somitogenesis Using Multiphoton Laser Scanning Microscopy (MPLSM)
}

\author{
Mary E. Dickinson ${ }^{\mathrm{a}^{* *}}$, Kenneth J. Longmuir ${ }^{\mathrm{b}}$ and Scott E. Fraser ${ }^{\mathrm{a}}$ \\ ${ }^{a}$ Biological Imaging Center, Beckman Institute, Caltech, Pasadena, CA 91125 \\ ${ }^{b}$ Department of Physiology \& Biophysics, College of Medicine, University of California, \\ Irvine, CA 92697-4560.
}

\begin{abstract}
In order to study complex cellular interactions in the developing somite and nervous system, we have been refining techniques for labeling and imaging individual cells within the living vertebrate embryo. Most recently, we have been using MPLSM to analyze cellular behaviors, such as cell migration, filopodial extension, cell process collapse, and neuron pathfinding using time-lapse microscopy in 3-dimensions (3-d). To enhance the efficiency of two-photon excitation in these samples, we have been using a Zeiss LSM 510 NLO fiber delivery system with a Grating Dispersion Compensator (GDC). This system not only offers the convenience of fiber delivery for coupling our Ti:Sapphire laser to the microscope, but also affords us precise control over the pulsewidth of the mode-locked beam. In addition, we have developed a novel peptide/non-cationic lipid gene delivery system to introduce GFP plasmid into somite cells. This approach has allowed us to generate detailed 3-d images of somite cell morphologies at various stages of somite development in a way that best preserves the vitality of the cells being imaged.
\end{abstract}

Keywords: Multiphoton microscopy, GFP, Somitogenesis, liposomes, filopodia, optical fibers

\section{INTRODUCTION}

The vertebrate somites are transient mesodermal structures that form on either side of the neural tube during early development. Cells from the somite contribute to a variety of derivatives

\footnotetext{
${ }^{*}$ Correspondence: Email: maryd@gg.caltech.edu
} 
in the mature animal, including components of the axial skeleton, skeletal musculature and dermis of the skin. Somitogenesis begins in the axial mesoderm as loosely associated mesenchymal cells coalesce to form epithelial blocks of tissue. Over the course of somite development, the epithelial blocks undergo considerable changes in response to signals from the environment (see ${ }^{1}$ for review). During differentiation, the epithelial block of tissue is divided into a sclerotome and a dermomyotome population. Sclerotome cells will delaminate and migrate to contribute to the axial skeleton, while dermomyotome cells will split into two populations, one that will contribute to the dermis of the skin, and another to the skeletal musculature. Thus, the differentiation of somitic cells involves many dynamic events as cells undergo a mesenchymal to epithelial to mesenchymal cell transition. Our group is interested in the studying the changes in cell behavior that occur during somite development, in order to understand how particular cell signaling events orchestrate these changes.

For our characterization of somite development, we have begun to collect highresolution, three-dimensional (3-d) images of cell morphologies at various stages of chick development using Multiphoton Laser Scanning Microscopy (MPLSM). MPLSM has emerged as an important technical advancement for vital and deep tissue imaging. In MPLSM, fluorescent molecules are excited using pulsed IR light that can penetrate into the tissue deeper than visible range light and causes less photodamage. Moreover, this mode of excitation has a quadratic dependence, producing excitation only at the focal plane; thus, out-of-focus fluorescence does not contribute to image background and photodamage outside the plane of focus is eliminated. The high signal-to-noise ratios achieved using this technique enhances our ability to examine fine structural details in the somite tissue.

For these studies, we have taken advantage of two technological advances to aid in our study. First, we have been using a state-of-the-art Zeiss 510 NLO system with fiber-delivery and 
pulse-width compensation to obtain images of the developing somite. This system allows us to achieve efficient excitation of the fluorochrome with minimal power. Second, we have used a novel, multi-component, lipid transfection system to introduce plasmid expressing Green Fluorescent Protein (GFP) into the cells of the pre-somitic mesoderm. GFP is a very bright fluorescent marker with uniform expression and is ideal for MPLSM. Thus, the combination of these approaches provides us with a way to introduce GFP plasmid into pre-somitic mesoderm cells and to analyze the morphologies of those cells soon after they coalesce into an epithelial somite.

\section{MATERIALS AND METHODS}

\subsection{Zeiss LSM 510 NLO fiber delivery system}

Our Zeiss 510 NLO system was obtained from Carl Zeiss, Inc. (Thornwood, NY). The Mira 900F laser system, including a 5W pump laser (Coherent Lasers, Inc., Santa Clara, CA) was aligned with a Grating Dispersion Compensator (GDC) that was provided by Coherent Lasers, Inc. (Santa Clara, CA). A Zeiss Acousto-optic modulator (Carl Zeiss, Jena, Germany) was placed inside the GDC before the fiber mount. The fiber was provided by Coherent Lasers, Inc. (Santa Clara, CA), as was the Post Fiber Compression (PFC) unit. All items listed above were installed and aligned with the help of application engineers from both Carl Zeiss, Inc. and Coherent Lasers, Inc.

\subsection{GFP expressing plasmid}

A 5757 bp plasmid containing green fluorescent protein (GFP) reporter gene with a human cytomegalovirus (CMV) immediate early gene promoter/enhancer region $\left(\mathrm{pWIZ}^{\mathrm{TM}}\right)$ was purchased from Gene Therapy Systems (San Diego, CA).

\subsection{Dye labeling}

Both DiA and Hoescht 33342 were purchased from Molecular Probes, Inc. (Eugene, OR). DiA was dissolved at $5 \mathrm{mg} / \mathrm{ml}$ in $\mathrm{EtOH}$ and a bolus of this solution was injected into the neural tube adjacent to the GFP labeled somite. DiA injections were performed while the embryos were 
still in the egg after confirmation of GFP labeling at 12 and 24 hours. For Hoescht 33342

labeling, embryos were extracted from the egg and soaked in a 1:1000 dilution of the standard stock $(10 \mathrm{mg} / \mathrm{ml})$.

\subsection{Components of the lipid transfection system}

Details of the lipid transfection system can be found in ${ }^{2,3}$. An optimized formulation was used here and consisted of a 4.25:1 ratio of positive charge on the condensing peptide to negative charge on the plasmid DNA, $100 \mathrm{nmol}$ if lipid per $10 \mathrm{~g}$ of plasmid DNA, a 15:1 ratio of lipid to fusogenic peptide and $20 \%$ polyethylene glycol.

\subsection{Analysis of somite cells in chick embryos}

For experiments to examine fine structures in embryos, fertile, white leghorn chicken eggs (AA labs, Westminster, CA) were incubated at 37 degrees in approx. 50\% relative humidity for $50-56$ hours to obtain Stage 14-16 embryos ${ }^{4}$. Green Fluorescent Protein expressing plasmid was introduced into the presomitic mesoderm of the embryo by injecting the DNA complexed in with components of the novel multicomponent lipid transfection system ${ }^{2,3}$ into the loose, unsegmented mesoderm on the right side of the neural tube. Embryos were analyzed 12, 24 and 48 hours post-injection of the lipid:DNA complex.

\subsection{Image collection and post-processing}

For imaging, all embryos were removed from the egg, washed in PBS and placed on a microscope slide. Black electrical tape was used to make a spacer so that the weight of the coverslip would not harm the embryo. All images were collected using Zeiss 510 LSM software (Version 2.5 and 2.8) and processed using the advanced 3-D functions in the Zeiss 510 LSM Version 2.8 software (Carl Zeiss, Inc, Thornwood, NY). Details of each image and post-processing are given in the figure legends for each image shown.

\section{RESULTS AND DISCUSSION}

\subsection{Pulsewidth compensation for fiber delivery}

Our system consists of an upright Zeiss 510 confocal microscope coupled to a Coherent Mira 900 Ti:Sapphire laser via a 3 meter fiber. Optical fibers are not routinely used for MPLSM because Group 
Velocity Dispersion (GVD) results in a significant broadening of the pulse as it passes through the fiber. At $800 \mathrm{~nm}$, a $100 \mathrm{fs}$ pulse with a bandwidth of $10 \mathrm{~nm}$ passing through a 3 meter fiber will be stretched into the ps range. This is because the fiber imparts a positive chirp on the pulse. Red light travels faster through this dispersive media than blue light so the pulse gets expanded. Recently, Wolleschensky et al. ${ }^{5}$ have shown that fiber delivery is possible for MPSLM. We have employed a similar compensation system manufactured by Coherent Lasers, Inc (Santa Clara, CA). To compensate for GVD through the fiber as well as dispersive effects from other elements in the scanhead and the microscope, the pulse is passed through a Grating Dispersion Compensator (GDC) to impart a negative chirp on the pulse before it enters the proximal end of the fiber. This device, combined with a Post Fiber Compression (PFC) unit placed between the distal end of the fiber and the scanhead, results in a 120 to $200 \mathrm{fs}$ pulse at the sample ${ }^{6}$. However, one caveat of this system is that the use of the fiber limits the overall power of the pulses delivered to the microscope. The fiber we use has a capacity of $<65 \mathrm{~mW}$. More power through the fiber will result in self-phase modulation. We have measured power levels of $3-45 \mathrm{~mW}$ at the sample, depending on the obective lens that is used ${ }^{6}$. In most cases, this has been sufficient to generate large, high quality 3-d data sets from embryonic tissue deep in the embryo (see below). For instance, one data set that is partially shown in Figure 1C, consisted of images taken from the surface down to $146 \mathrm{~m}$ into the embryo. In addition, we have succeeded in collecting high quality images of cornea tissue $850 \mathrm{~m}$ deep in the sample (data not shown).

\subsection{Transfection of chick somite cells in vivo}

While GFP expression constructs can be introduced into chick embryos by viral infection or electroporation of plasmid DNA, both methods have drawbacks. Neither method is capable of limiting plasmid expression to a defined area; furthermore, cells infected by retroviral constructs do not have enough GFP expression for imaging until at least 24 hours after infection ${ }^{7}$. For these reasons, we sought to identify alternative ways to introduce GFP expressing plasmids into localized regions of the pre-somitic mesoderm. Toward this end, we have developed a novel, multi-component lipid transfection system to introduce GFP expression constructs into early somite cells ${ }^{2,3}$. In addition to plasmid DNA, the delivery complex consists of four components: 1) a condensing peptide, 2) a fusogenic peptide, 3) a relatively 
short-chain phosphatidylcholine (14:1 cis-9), and 4) polyethyleneglycol conjugated to

dioleoylphosphatidylethanolamine through a disulfide linkage. Through a systematic analysis of component concentrations, we have developed an optimized formulation for plasmid delivery into chick cells in vitro and in vivo ${ }^{3}$. This method provides an easy and quick way to introduce plasmid DNA since the lipid complexes can be concentrated to $>1 \mathrm{mg}$ plasmid DNA per $\mathrm{ml}$ for localized nanoliter delivery via injection. GFP expression is seen in these cells as soon as 6 hours post-injection, and by 12 hours, many bright cells are evident in the injected area (data not shown).

For the experiments shown here, we injected a small bolus of the multi-component transfection reagent containing GFP expression plasmid into the presomitic mesoderm of Stage 12-14 chick embryos 2 . As previously described ${ }^{3}$, we have developed an optimized formulation for transfection of chick cells. Twenty-one embryos were injected with this formulation and analyzed at various times for GFP expression. GFP positive cells were found in 4 of 4 embryos examined twelve hours after injection, 13 of 13 embryos examined twenty-four hours after injection and 4 of 4 embryos examined forty-eight hours after injection. Thus, $100 \%$ of the embryos injected with the formulation contained cells transfected with GFP plasmid. Although we have not fully characterized the number of cells transfected at each injection site, the quantity of GFP positive cells ranged from ten cells to hundreds of cells (data not shown).

\subsection{Analysis of GFP-labeled cells}

Using our fiber-delivery MPLSM system, we have analyzed transfected cells 12, 24 and 48 hours post-injection. At all stages of development, we have been able to identify normal somite cell morphologies (Figure 1). In younger embryos, 12-24 hours after injection, labeled somite cells have close associations with other cells within epithelial structures (Figure 1A and B). In older somites, 48 hours after injection, mature cell types can be easily identified. In particular, myotubes have begun to form and are evident as elongated parallel structures within the injected region (Figure 1C). These data are consistent with evidence collected using histological methods (data not shown). 
Interestingly, in all embryos examined at all stages, we have been able to identify cells that extend fine cellular extensions (Figure 1A, B,and C). These projections often extend toward and contact other cells. Pattern formation in the somite is regulated through a series of cell signaling events ${ }^{1}$. Signals from both the neural tube and the overlying are thought to influence somite differentiation ${ }^{1}$. Thus, we have begun to investigate whether these filopodial extensions could play a role in cell-cell communication between the somite and the neural tube and/or the overlying ectoderm.

In order to be able to identify neural tube cells and cells in the overlying ectoderm, we have used DiA, a lipophilic carbocyanine dye, to label the neural tube and Hoescht 33342 to stain cells in the overlying ectoderm. DiA can easily be injected into the neural tube adjacent to labled somite cells. This dye is quickly incorporated into the cell membranes and does not pass through the tube into surrounding tissues. Hoescht 33342 is applied by injecting it or soaking the embryo in the dye. The dye does not penetrate well into the live embryo, but labels cells in the surface ectoderm and cells close to the surface. Used together with GFP labeling, it is possible to determine whether somite cells are contacting the neural tube or the overlying ectoderm.

In our initial analysis, we counterstained five embryos using this technique, two embryos from the 12 hour post-injection group and 3 from the 24 hour post-injection group. In one embryo from the $12 \mathrm{~h}$ group and 2 embryos from the $24 \mathrm{~h}$ group, we found evidence of contact between the neural tube and/or the surface ectoderm. Images from two of these embryos are shown in Figure 2. In Figure 2A, GFP expressing cells in a 12 hour post-injection embryo have extended filopodia toward the neural tube, whereas in figure 2B somite cells have extended filopodia toward the cell surface.

These preliminary data show that cell-cell contacts between somite cells and other nearby tissues are established early in development. Pattern formation in the somite is thought to be regulated by signals secreted by cells in nearby tissues ( see $^{1}$ for review). These studies suggest that direct contact through cell surface molecules could also be involved in pattern formation. In future studies we plan to investigate how filopodial extensions are regulated and if cell-cell contact is necessary for proper somite development. 


\section{REFERENCES}

1. Dockter, J.L. Sclerotome Induction and Differentiation. Pp 71-127. In, Current Topics in Developmental Biology, Vol. 48: Somitogenesis (Part 2). C. P. Ordahl, Ed. Academic Press, San Diego, 2000.

2. Longmuir K.J., Murphy, E.A., Waring, A.J., Dickinson, M.E. and Haynes, S.M. A Nonviral Liposomal Complex Designed to Overcome the Multiple Barriers to

Gene Transfer. Molecular Therapy 5, S243, 2000.

3. Longmuir, K.J., Haynes, S.M., Dickinson, M.E., Waring, A.J. and Murphy, J.C. Optimization of a peptide/non-cationic lipid gene delivery system for effective microinjection into chicken embryo in vivo. Mol. Therapuetics, submitted.

4. Hamburger, V. and Hamilton, H.L. A series of normal stages in the development of the chick. J. Morph. 88, 48-92, 1951.

5. Wolleschensky, R., Feurer T., Sauerbrey R., and Simon I. Characterization and optimization of a laserscanning microscope in the femtosecond regime. Applied Physics B-Lasers and Optics 67, 87-94, 1998.

6. M. Dickinson, R. Wolleschensky and S. Fraser, unpublished observations.

7. Okada, A., Lansford, R., Weimann, J.M, Fraser, S.E. and McConnell, S.K. (1999) Imaging cells in the Developing Nervous System with Retrovirus Expressing Modified Green Fluorescent Protein. Exp.

Neurology 156, 394-406.
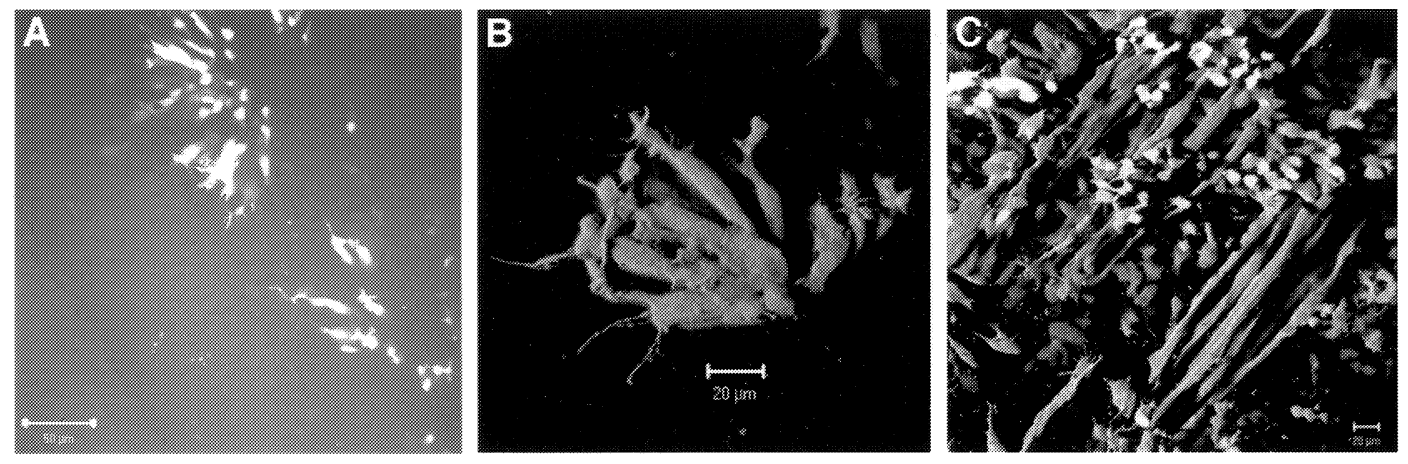

Figure 1. GFP lableled cells in the chick embryo at 24 (A,B) and 48 (C) hours post-injection. In A and B, GFP labeled cells are arranged within the somite epithelium. At 48 hours, differentiated cells are labeled with GFP. In particular, elongated myotubes have begun to form amidst other labeled cells. A is a single confocal image taken using $488 \mathrm{~nm}$ excitation and a LP 505 emission filter. This is a multi-channel image and is superimposed on the transmitted light image taken using Nomarski optics. B and C are two-photon images using $860 \mathrm{~nm}$ excitation and a short pass $680 \mathrm{~nm}$ emission filter. Both are 3-D renderings (shadow rendering in the Zeiss 510 LSM software v. 2.8) of stacks of images taken at $0.5 \mu \mathrm{m}$ intervals. Both stacks initially consisted of 140 and 292 images, respectively, but smaller subsets of the data were used to make the projections shown here (120 \& 104 images, respectively). For both images, the threshold was set at 7 . In all cases, filopodial extensions can be observed coming from labeled somite cells. 

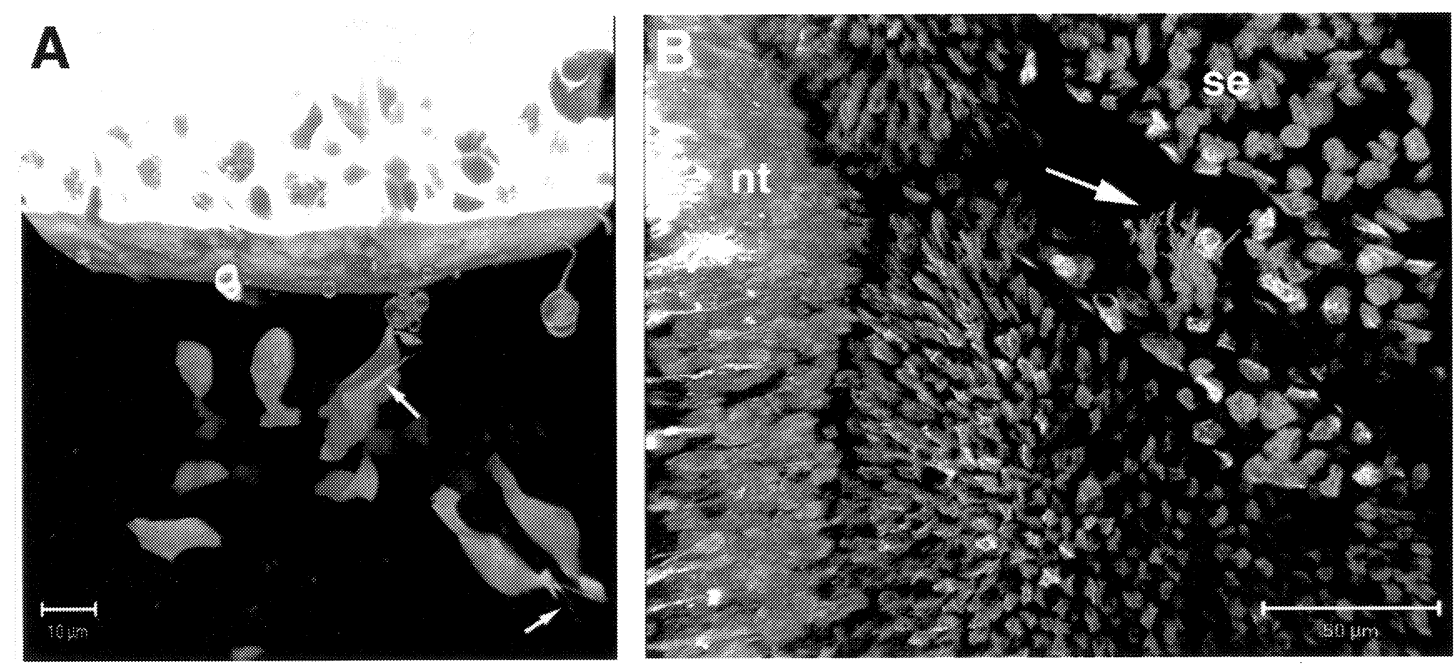

Figure 2. GFP labeled cells in the context of other labeled cells. A shows data from an embryo imaged 12 hours postinjection of the lipid:DNA complex. The solid mass of cells at the tope of the image are the neural tube cells labeled with $\mathrm{DiA}$, whereas individual cells in the lower half of the figure are labeled with GFP. This transparency rendering (threshold=4) was made in the Zeiss 510 LSM v. 2.8 software and consists of 166 images taken at $0.5 \mu \mathrm{m}$ intervals. C is also a transparency rendering of 102 optical slices taken at $0.5 \mu \mathrm{m}$ intervals (threshold=6). GFP cells can be seen extending filopodia toward surface ectoderm (se) cells that are labeled with Hoescht 33342. The neural tube (nt) is labeled with DiA. Many, long filopodia (arrows) can be observed extending toward the neural tube or the surface ectoderm. 